\title{
A Turntable-based Approach for Ground Truth Tracking Data Generation
}

\author{
Zoltán Pusztai $^{1,2}$ and Levente Hajder ${ }^{1}$ \\ ${ }^{1}$ Distributed Events Analysis Laboratory, MTA SZTAKI, Kende utca 13-17. H-1111, Budapest, Hungary \\ ${ }^{2}$ Eötvös Loránd University, Budapest, Hungary \\ hajder.levente@sztaki.mta.hu,puzsaai@inf.elte.hu
}

Keywords: Ground Truth Dataset, Ground Truth Dataset, 3D Scanner, 3D Reconstruction.

\begin{abstract}
Quantitative evaluation of feature trackers can lead significant improvements in accuracy. There are widely used ground truth databases in the field. One of the most popular datasets is the Middlebury database to compare optical flow algorithms. However, the database does not contain rotating 3D objects. This paper proposes a turntable-based approach that fills this gap. The key challenge here is to calibrate very accurately the applied camera, projector, and turntable. We show here that this is possible, even if just a simple chessboard plane is used for the calibration. The proposed approach is validated on 3D reconstruction and ground truth tracking data generation of real-world objects.
\end{abstract}

\section{INTRODUCTION}

Developing a realistic 3D approach for feature tracker evaluation is very challenging since realistic moving 3D objects can simultaneously rotate and translate, moreover, occlusion can also appear in the images. It is not easy to implement a system that can generate ground truth (GT) data for real-world 3D objects. The aim of this paper is to present a novel structured-light reconstruction system that can produce extremely accurate feature points of rotating spatial objects.

The Middlebury database ${ }^{1}$ is considered as the state-of-the-art GT feature point generator. The database itself consists of several datasets that had been continuously developed since 2002. In the first period, they generated corresponding feature points of real-world objects (Scharstein and Szeliski, 2002). The first Middlebury dataset can be used for the comparison of feature matchers. Later on, this stereo database was extended with novel datasets using structured-light (Scharstein and Szeliski, 2003) or conditional random fields (Pal et al., 2012). Even subpixel accuracy can be achieved in this way as it is discussed in (Scharstein et al., 2014).

However, our goal is to generate tracking data via multiple frames, the stereo setup is too strict limitation for us.

\footnotetext{
${ }^{1}$ http://vision.middlebury.edu/
}

The description of the optical flow datasets of Middlebury database was published in (Baker et al., 2011). It was developed in order to make the optical flow methods comparable. The latest version contains four kinds of video sequences:

1. Fluorescent Images: Nonrigid motion is taken by a color and a UV-camera. Dense ground truth flow is obtained using hidden fluorescent texture painted on the scene. The scenes are moved slowly, at each point capturing separate test images in visible light, and ground truth images with trackable texture in UV light.

2. Synthesized Database: Realistic images are generated by an image syntheses method. The tracked data can be computed by this system as every parameters of the cameras and the $3 \mathrm{D}$ scene are known.

3. Imagery for Frame Interpolation: GT data is computed by interpolating the frames. Therefore the data is computed by a prediction from the measured frames.

4. Stereo Images of Rigid Scenes: Structured light scanning is applied first to obtain stereo reconstruction. (Scharstein and Szeliski 2003). The optical flow is computed from ground truth stereo data.

The main limitation of the Middlebury optical flow database is that the objects move approximately 
linearly, there is no rotating object in the datasets. This is a very strict limitation as tracking is a challenging task mainly when the same texture is seen from different viewpoint.

It is interesting that the Middlebury multi-view database (Seitz et al., 2006) contains ground truth 3D reconstruction of two objects, however, the ground truth tracking data were not generated for these sequences. Another limitation of the dataset is that only two low-textured objects are used.

It is obvious that tracking data can also be generated by a depth camera (Sturm et al., 2012) such as Microsoft Kinect, but its accuracy is very limited. There are other interesting GT generators for planar objects such as the work proposed in (Gauglitz et al., 2011), however, we would like to obtain the tracked feature points of real spatial objects.

Due to these limitations, we decided to build a special hardware in order to generate ground truth data. Our approach is based on a turntable, a camera, and a projector. They are not too costly, however, the whole setup is extremely accurate as it is shown here.

Accurate Calibration of Turntable-based 3D Scanners. The application of structured-light scanner is a relatively cheap and accurate possibility to build a real 3D scanner as it is discussed in the latest work of (Moreno and Taubin, 2012). Another possibility for a very accurate 3D reconstruction is laser scanning (Bradley et al., 1991), however, the accurate calibration of the turntable is not possible using a laser stripe since it can only reconstruct a $2 \mathrm{D}$ curve at a moment. For turntable calibration, the reconstruction of $2 \mathrm{D}$ objects is a requirement since the axis of the rotation can be computed by registrating the point clouds of the same rotating object.

Moreover, the calibration of the camera and projector intrinsic and extrinsic parameters is also crucial. While the camera calibration can be accurately carried out by the well-known calibration method of (Zhang, 2000), the projector calibration is a more challenging task. The projector itself can be considered as an inverse camera: while the camera projects the $3 \mathrm{D}$ world to the $2 \mathrm{D}$ image, the projector projects the planar image onto the 3D world. For this reason, the corresponding points of the $3 \mathrm{D}$ world and the projector image cannot be matched. Therefore, firstly the pixel-pixel correspondences have to be detected between the camera and the projector. The application of structured light was developed in order to efficiently realize this correspondence detection (Scharstein and Szeliski, 2003).

Many projector calibration methods exist in the field. The first popular class of existing solu- tions (Sadlo et al., 2005; Liao and Cai, 2008; Yamauchi et al., 2008) is to (i) use a calibrated camera to determine the world coordinate, (ii) then a pattern is projected onto the calibration plane, the corners are detected and locations are estimated in 3D, (iii) then the $3 \mathrm{D} \rightarrow 2 \mathrm{D}$ correspondences are given by running the (Zhang, 2000) calibration. The drawback of this kind of approaches is that its accuracy is relatively low since the projected 3D corner locations are estimated, and these estimated data are used for the final calculation.

Another possible solution is to ask the user to move the projector at different positions (Anwar et al., 2012; Jamil Draréni, 2009). It is not possible for our approach as the projector is fixed. Moreover, the accuracy of these kind of approaches is also very low.

There are algorithms where both projected and printed pattern are used (Audet, 2009; Martynov et al., 2011). The main idea here is that if the projected pattern is iteratively adjusted until it fully overlaps the printed pattern, then the projector parameters can be estimated. Color patterns can also be applied for this purpose (Park and Park, 2010). However, we found that this quite complicated method is not required to calibrate the camera-projector system.

Our calibration methods for both the camera and projector use a simply chessboard plane. Our algorithms are very similar to those of (Moreno and Taubin, 2012). As it is shown here later, we calibrate the camera first by the method of (Zhang, 2000). Then the point correspondences between camera and projector pixels are determined by robustly estimating the local homography close to the chessboard corners. Then the intrinsic projector parameters can be computed by (Zhang, 2000) as well. The extrinsic parameters (relative translation and orientation between the camera and the projector) can given by a stereo calibration problem. For this purpose, there are several solutions as it is discussed in (Hartley and Zisserman, 2003) in detail. However, we found that the accuracy of stereo calibration is not accurate, therefore we proposes a more sophisticated estimation here.

Contribution of this Study. The main novelty of this paper is that we show here that very accurate GT feature data can be generated for rotating object if a camera-projector system is applied with turntable. To the best of our knowledge, our approach is the first system that can yield such accurate GT tracking data. The usage of a turntable for $3 \mathrm{D}$ reconstruction itself is not a novel idea, but its application for GT data generation it is.

The calibration algorithms within the system have a minor and a major improvements:

- The camera-projector correspondence estimation 

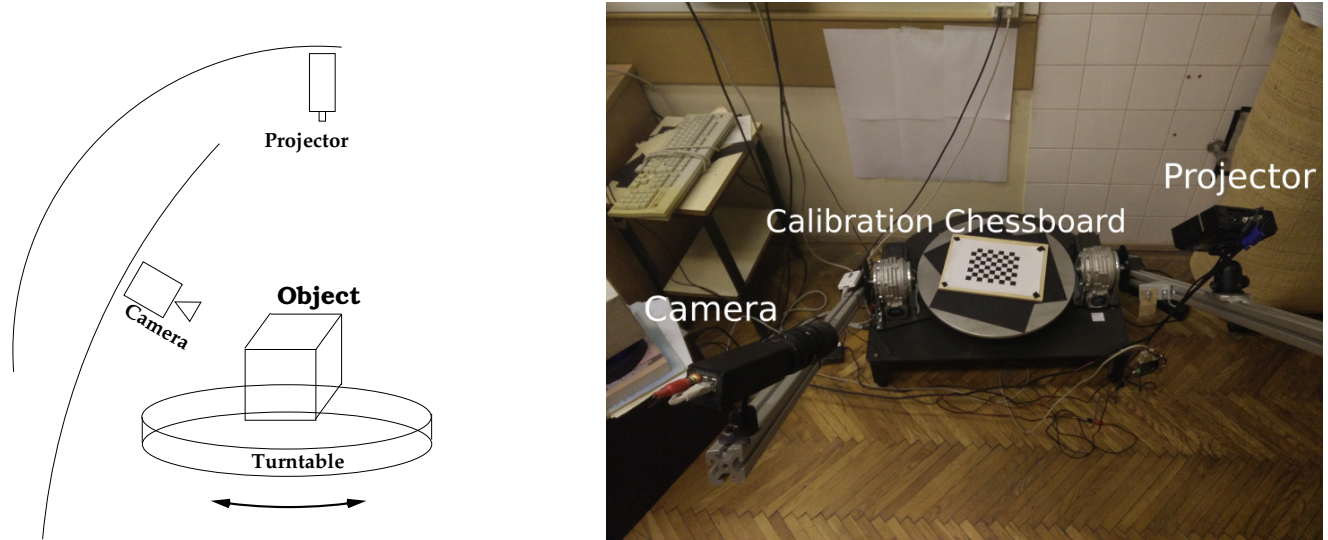

Figure 1: Hardware components of structured light scanner. Left: schematic figure. Right: Realized scanner.

is based on a robust (RANSAC-based) homography estimation.

- The turntable calibration is totally new: while usual turntable calibrators (Kazo and Hajder, 2012) compute the axis by performing a usual chessboard-based calibration method (Zhang, 2000) for the rotating chessboard plane, and the axis of the rotation is computed from the extrinsic camera parameters, we propose a novel optimization problem that minimizes the reprojection for the corners of the rotating chessboard. We found the accuracy of this novel algorithm is significantly better. During the turnable calibration, the extrinsic parameters of the camera and projector are also obtained.

\section{PROPOSED EQUIPMENT AND ALGORITHMS}

Our 3D scanner consists of 3 main parts. It is visualized in Fig. 1. The left plot is the schematic setup, while the right one shows the realization of the scanner. The main components of the equipment are the camera, the projector, and the turntable. Each of the above needs to be calibrated correctly to reach high accuracy in 3D scanning. The camera and the projector are fixed to their arms, but the turntable can move $^{2}$ : it is able to rotate the object to be reconstructed.

The bottleneck of the proposed approach is the calibration of the components. In this section, it is overviewed how the camera, the projector, and the axis of the rotating table can be accurately calibrated.

\footnotetext{
${ }^{2}$ These arms are also moving, but their calibration is not considered here, it is a possible future work.
}

The paper is organized as follows. The software components are overviewed in Figure 2. The camera, projector and turntable calibration is described in Section 2.1, 2.1, and 2.3, respectively. Sec. 3 shows how accurate GT data can be generated by the developed equipment. Finally, Sec. 6 concludes the work and discusses the limitations. calibration,

\subsection{Camera Calibration}

For describing the camera we choose the pinhole model with radial distortion. Assuming that the coordinate system is aligned to the camera, the projection of the point $X \in \mathbb{R}^{3}$ to the camera plane is $u \in \mathbb{R}^{2}$, which can be described by the equation:

$$
\begin{gathered}
u=K_{c} \tilde{u}, \\
K_{C}=\left[\begin{array}{ccc}
f_{x} & \gamma & p_{x} \\
0 & f_{y} & p_{y} \\
0 & 0 & 1
\end{array}\right], \\
\tilde{u}=\left[\begin{array}{l}
u_{x}\left[1+k_{1} r^{2}+k_{2} r^{4}\right] \\
u_{y}\left[1+k_{1} r^{2}+k_{2} r^{4}\right]
\end{array}\right], \\
r^{2}=u_{x}^{2}+u_{y}^{2},
\end{gathered}
$$

where $K_{C}$ stands for the camera matrix, $f_{x}$ and $f_{y}$ are the focal length, $\left(p_{x}, p_{y}\right)$ is the principal point, and $\gamma$ is the shear. In our case we only used radial distortion, which can be described by two parameters: $\left(k_{1}, k_{2}\right)$. The camera matrix and the distortion parameters are together called the intrinsic parameters of the camera.

A black and white chessboard is held in sight of the camera at arbitrary positions. Images were taken and the chessboard corners were found on the images, and they refined to reach sub-pixel precision. Then we can compute the intrinsic parameters of the camera by the method of (Zhang, 2000). 


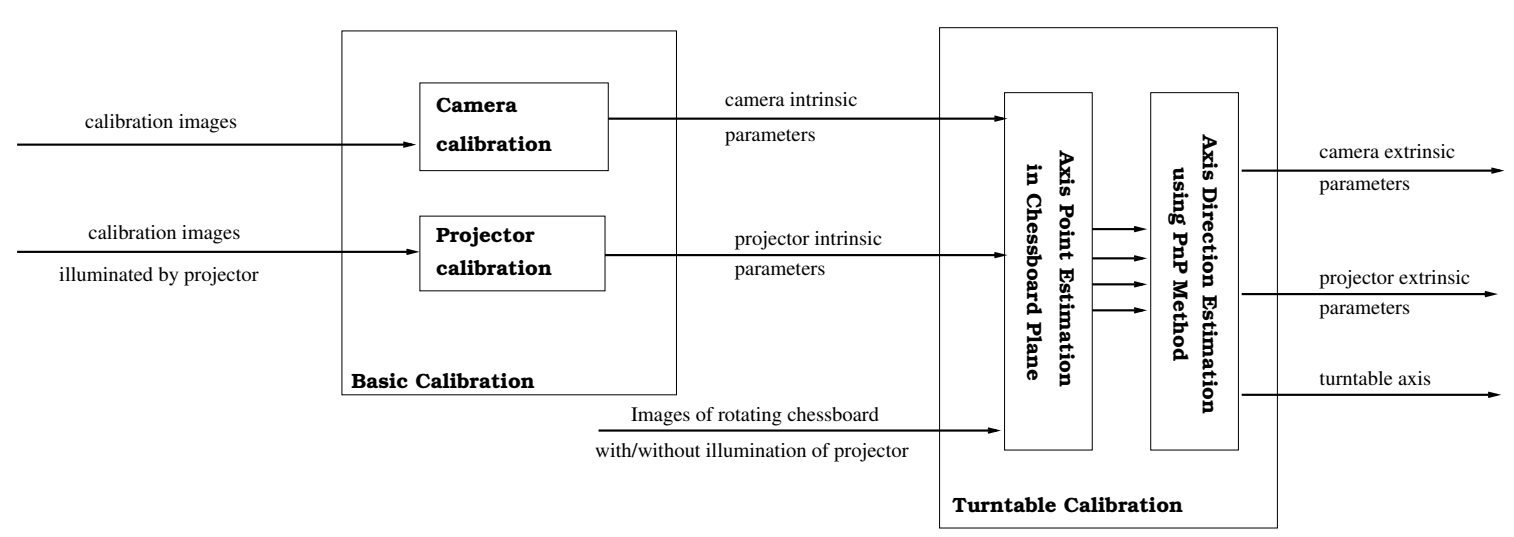

Figure 2: Software components of the whole calibration pipeline.

\subsection{Projector Calibration}

Since the projector can be viewed as an inverse camera, it can be described by the same model applied for the camera before. However, finding the right projector pixels, which through the chessboard corners are seen from the viewpoint of the projector is not so obvious. To overcome this problem, a structured light sequence is projected to the scene. It precisely encodes the pixel coordinates in the projector image. For each scene point, the projected codes has to be decoded. From this point, the chessboard must be placed in a position that can be viewed from both the camera and projector.

The structured light we used for the calibration is based on the binary Gray code since it is the most accurate coding for structured light scanning as it is discussed in (Scharstein and Szeliski, 2003). In addition, we project inverse images after every single one, meaning that every pixel on the images is reversed. But before structured light utilized, full black and white images are projected for easier object recognition, and for easier decoding of the structured light.

Since the resolution of our projector is $1024 \times 768$, the number of the projected images are 42 for each chessboard orientation. The projected sequence consists of 2 pure black and white images, 10 images for encoding the horizontal, and 10 for encoding the vertical coordinates of each projector pixel. Additionally, the inverse images have to be inserted into the sequence as well. These images are taken from one viewpoint, and they are called as the image set.

After all the images are taken, one can begin the decoding of the structured light. First of all, we calculate the direct and indirect intensity of the light, pixel by pixel for each image set. The full method is described in (Nayar et al., 2006). Then the minimum and maximum intensities are determined per pixel and then the direct and indirect values are given by the equations as follows:

$$
\begin{gathered}
L_{D}=\frac{L_{\text {max }}-L_{\text {min }}}{1-B} \\
L_{I}=\frac{2\left(L_{\text {min }}-B * L_{\text {max }}\right)}{1-B^{2}}
\end{gathered}
$$

Where $B$ is the amount of light emitted by a turned-off projector pixel. We needed to separate these 2 components from each other, because we are only interested in the direct intensities lit by the projector.

Then we need to classify the pixels on each image pair, consisting the image given by the structured light and its inverse. There are 3 clusters to classify into:

1. The pixel is lit on the first image.

2. The pixel is not lit on the first image.

3. Cannot be determined.

The classification rules are as follows:

- $L_{D}<M \Longrightarrow$ the pixel is in the 3. class,

- $L_{d}>L_{I} \wedge P_{1}>P_{2} \Longrightarrow$ the pixel is lit,

- $L_{d}>L_{I} \wedge P_{1}<P_{2} \Longrightarrow$ the pixel is not lit,

- $P_{1}<L_{D} \wedge P_{2}>L_{I} \Longrightarrow$ the pixel is not lit,

- $P_{1}>L_{I} \wedge P_{2}<L_{I} \Longrightarrow$ the pixel is lit,

- otherwise it cannot be determined.

The pixel intensity in the first and inverse images are denoted by $P_{1}$, and $P_{2}$, respectively, while $M$ is a userdefined threshold: $M=5$ is set in our approach. If the difference between $P_{1}$ and $P_{2}$ is greater than $M$, then the pixel is discarded.

For further reading about the classification, we recommend to read the study of (Xu and Aliaga, 2007).

Since the chessboard consists of alternating black and white squares, decoding near the chessboard corners can resolve errors. To avoid these errors, we calculate local homographies around the corners. We use 
11 pixel-wide kernel window and every successfully decoded projector pixel is in consideration. For the homography estimation, a RANSAC-based (Fischler and Bolles, 1981) DLT homography estimator is applied in contrast to the work of (Moreno and Taubin, 2012) where robustification is not dealt with. We found that the accuracy is increased when RANSACscheme is applied. After the homography is computed among the camera and projector pixels, we use this homography to transform the camera pixels to the projector image. In this way we get the exact projector pixels we needed, so we can use the method of (Zhang, 2000) to calibrate the projector. Remark that the extrinsic projector calibration will be refined later, but the intrinsic parameters will not.

\subsection{Turntable Calibration}

The aim of the turntable calibration is to compute the axis of the turntable. It is represented by a point and a direction. Therefore, the degree of freedom of a general axis estimation is four (2 DoFs: position of a plane; other 2 DoFs: direction) .

Fortunately, the current problem is constrained. We know that the axis is perpendicular to the plane of the turntable. Thus, the direction is given, only the position should be calculated within the turntable plane.

The turntable is calibrated if we know the centerline which the table is turning around. Two methods was used to calculate this 3D line. First we place the chessboard on the turntable, and start rotating it. Images are taken between the rotations, and the extrinsic parameters can be computed for each image since the camera is already calibrated. This motion is equivalent with the motion of a steady chessboard and a moving camera. The circle that the camera follows has the same centerline as the turntable. Thus fitting a circle to the camera points estimates the centerline of the turntable (Kazo and Hajder, 2012).

However, we found that this method is not accurate enough. Therefore, we developed a novel algorithm that is overviewed in the rest of this section.

\subsubsection{Problem Statement of Turntable Axis Calibration}

Given a chessboard with known size, for which the corners can be easily detected by widely used pattern recognition algorithms, the goal is to estimate the axis of the turntable. This is part of the calibration of a complex structured-light 3D reconstruction system that consists of one camera, one projector, and one turntable. The latter one is driven by a stepping motor, the angle of the rotation can be very accurately set. The camera and projector intrinsic parameters are also known, in other words, they are calibrated.

The input data for axis calibration comes from detected chessboard corners. The chessboard are placed on the turntable. Then it is rotated and images are taken with different rotational axis. The corners are detected on all of these images. Then the chessboard is placed in a higher position on the turntable, but the new plane orientation is also parallel to the turntable. Then the chessboard are rotated, and the corners are detected as well. (The chessboard can be placed in arbitrary altitudes. We only use two different values, but the proposed calibration method can work with arbitrary number of positions.)

If we consider the case when the planes of the chessboard and the turntable are parallel, the distance between them is $h$, then the chessboard corners can be written as

$$
\begin{array}{r}
{\left[\begin{array}{l}
X \\
Y
\end{array}\right]=} \\
{\left[\begin{array}{cc}
\cos \alpha & -\sin \alpha \\
\sin \alpha & \cos \alpha
\end{array}\right]\left[\begin{array}{c}
x-o_{x} \\
y-o_{y}
\end{array}\right]+\left[\begin{array}{c}
o_{x} \\
o_{y}
\end{array}\right]=} \\
{\left[\begin{array}{c}
\cos \alpha x-\sin \alpha y+o_{x}(1-\cos \alpha)+o_{y} \sin \alpha \\
\sin \alpha x+\cos \alpha y-o_{x} \cos \alpha+o_{y}(1-\cos \alpha)
\end{array}\right]}
\end{array}
$$

where $\alpha$ denotes the current angle of the rotation. Note that altitude $h$ does not influence the relationship. Also remark that capital $X$ and $Y$ denote spatial coordinates, while their lowercase letters ( $x$ and $y$ ) are $2 \mathrm{D}$ coordinates in image space.

\subsubsection{Proposed Algorithm}

The proposed axis calibration consists of two main steps:

1. Determination of the axis center $\left[o_{x}, o_{y}\right]^{T}$ on chessboard plane, and

2. computation of the camera and projector extrinsic parameters.

Axis Center $\left[o_{x}, o_{y}\right]^{T}$ Estimation on Chessboard Plane. The goal of the axis center estimation is to calculate the location $\left[o_{x}, o_{y}\right]^{T}$. We propose an alternation-type method with two substeps:

Homography-step. The plane-plane homography is estimated for each image. The 2D locations of the corners in the images are known. The 2D coordinates can be determined in the chessboard plane by Eq. 1. If the homogenous coordinates are used, the relationship becomes 
$H\left[\begin{array}{c}\cos \alpha x-\sin \alpha y+o_{x}(1-\cos \alpha)+o_{y} \sin \alpha \\ \sin \alpha x+\cos \alpha y-o_{x} \cos \alpha+o_{y}(1-\cos \alpha) \\ 1\end{array}\right]$.

We apply the standard normalized direct linear transformation (normalized DLT) with a numerical refinement step (Hartley and Zisserman, 2003) in order to estimate the homography. It solves the linearized version of Eq. 2:

$E\left(\alpha, x, y, o_{x}, o_{y}\right)=E_{1}\left(\alpha, x, y, o_{x}, o_{y}\right)+E_{2}\left(\alpha, x, y, o_{x}, o_{y}\right)$

where

$$
\begin{array}{r}
E_{1}\left(\alpha, x, y, o_{x}, o_{y}\right)= \\
u h_{31}\left(\cos \alpha x-\sin \alpha y+o_{x}(1-\cos \alpha)+o_{y} \sin \alpha\right)+ \\
u h_{32}\left(\sin \alpha x+\cos \alpha y+-o_{x} \cos \alpha+o_{y}(1-\cos \alpha)\right)+ \\
u h_{33}- \\
h_{11}\left(\cos \alpha x-\sin \alpha y+o_{x}(1-\cos \alpha)+o_{y} \sin \alpha\right)- \\
h_{12}\left(\sin \alpha x+\cos \alpha y+-o_{x} \cos \alpha+o_{y}(1-\cos \alpha)\right)- \\
h_{13}
\end{array}
$$

and

$$
\begin{array}{r}
E_{2}\left(\alpha, x, y, o_{x}, o_{y}\right)= \\
v h_{31}\left(\cos \alpha x-\sin \alpha y+o_{x}(1-\cos \alpha)+o_{y} \sin \alpha\right)+ \\
v h_{32}\left(\sin \alpha x+\cos \alpha y+-o_{x} \cos \alpha+o_{y}(1-\cos \alpha)\right)+ \\
v h_{33}- \\
h_{21}\left(\cos \alpha x-\sin \alpha y+o_{x}(1-\cos \alpha)+o_{y} \sin \alpha\right)- \\
h_{22}\left(\sin \alpha x+\cos \alpha y+-o_{x} \cos \alpha+o_{y}(1-\cos \alpha)\right)- \\
h_{13}
\end{array}
$$

This is a linear problem. The center and the scale of the applied coordinate system can be arbitrary chosen. As it is discussed in (Hartley and Zisserman, 2003), the mass center and quasi-uniform scale is the most accurate choice. The error function $E\left(\alpha, x, y, o_{x}, o_{y}\right)$ can be written for every chessboard corner point for every rotational angle. Therefore, the minimization problem is formulated as

$$
\arg \min _{H} \sum_{i=1}^{G_{x}} \sum_{j=1}^{G_{y}} \sum_{k=1}^{N} E\left(\alpha_{k}, x_{i, \alpha}, y_{j, \alpha}, o_{x, \alpha}, o_{y, \alpha}\right) .
$$

where $a_{k} \in[0,2 \pi], x_{i} \in\left[0, G_{x}\right]$, and $y_{i} \in\left[0, G_{y}\right]$, and $G_{x}, G_{y}$ are the dimensions of chessboard corners, respectively. (Possible values for $\left(x_{i}, y_{j}\right)$ are $(1,1),(1,2),(2,1), \ldots$ etc. ) This problem remains an over-constrained homogeneous linear one that can be optimally solved.

Axis-step. Its goal is to estimate the axis location $\left[o_{x}, o_{y}\right]^{T}$. The above two equations are linear with respect to the center coordinates. Therefore, the equations form a homogeneous linear system of equations $A\left[o_{x}, o_{y}\right]^{T}=b$, where

$$
A=\left[\begin{array}{ll}
a_{11} & a_{12} \\
a_{21} & a_{22}
\end{array}\right]
$$

where

$$
\begin{array}{r}
a_{11}=h_{11}-h_{11} \cos \alpha-h_{12} \sin \alpha- \\
u\left(h_{31}-h_{31} \cos \alpha-h_{32} \sin \alpha\right), \\
a_{12}=h_{11} \sin \alpha+h_{12}-h_{12} \cos \alpha- \\
u\left(h_{31} \sin \alpha+h_{32}-h_{32} \cos \alpha\right), \\
a_{21}=h_{21}-h_{21} \cos \alpha-h_{22} \sin \alpha- \\
v\left(h_{31}-h_{31} \cos \alpha-h_{32} \sin \alpha\right), \\
a_{22}=h_{21} \sin \alpha+h_{22}-h_{22} \cos \alpha- \\
v\left(h_{31} \sin \alpha+h_{32}-h_{32} \cos \alpha\right),
\end{array}
$$

and

$$
b=\left[\begin{array}{l}
b_{11}-b_{12} \\
b_{21}-b_{22}
\end{array}\right]
$$

where

$$
\begin{array}{r}
b_{11}=h_{13}+ \\
h_{11}(x \cos \alpha-y \sin \alpha)+h_{12}(y \cos \alpha+x \sin \alpha), \\
\left.b_{12}=h_{33}\right)+ \\
u\left(h_{31}(x \cos \alpha-y \sin \alpha)+h_{32}(y \cos \alpha+x \sin \alpha),\right. \\
b_{21}=h_{23}+ \\
h_{21}(x \cos \alpha-y \sin \alpha)+h_{22}(y \cos \alpha+x \sin \alpha), \\
b_{22}=h_{33}+ \\
v\left(h_{31}(x \cos \alpha-y \sin \alpha)+h_{32}(y \cos \alpha+x \sin \alpha)\right) .
\end{array}
$$

The above equations can be written for all corners of the chessboard for all rotated positions. Therefore, both the homography- and the axis-steps are extremely over-constrained. Thus, the parameters can be very accurately estimated. It is interesting that the homography and the axis location estimations are homogeneous, and inhomogeneous linear problems, respectively. They can also solved for the overdetermined case as it is well-known (Björck, 1996).

The two substeps have to be run one after the other. Both steps minimize the same algebraic error, therefore the method converges to the closest (local) minimum. Unfortunately, global optimum cannot be theoretically guaranteed. But we found that the algorithm converges to the correct solution. The speed of 
the convergence is relatively fast, to our experiments, $20-30$ iterations are required to reach the minimum. Parameter Initialization. The proposed alternation method requires initial values for $o_{x}$ and $o_{y}$. It has been found that the algorithm is not too sensitive to the locations of the initial values. The center of the chessboard is an appropriate solution for $o_{x}$ and $o_{y}$. Moreover, we have tried more sophisticated methods. If the camera centers are estimated by a Perspective n Point (PnP) algorithm such as (Lepetit et al., 2009), then the camera centers for the rotating sequence form a circle (Kazo and Hajder, 2012) as it is mentioned in the first part of this section. The center of this circle is also a good initial value. However, we found that the correct solution is reached as well if the initial center is an arbitrary point within the chessboard region.

\subsubsection{Axis Center Estimation in the Global System}

The first algorithm estimates the center of the axis in the coordinate system of the chessboard. But the chessboard are placed in different positions with different altitudes. The purpose of the algorithm discussed in this section is to place the rotated chessboard in the global coordinate system and to determine the extrinsic parameters (location and orientation) of the projector. The global system is fixed to the camera, therefore, the camera extrinsic parameters have not to be estimated.

In our calibration setup, only two chessboard sequences are taken. The extrinsic position can be easily determined. If the $3 \mathrm{D}$ coordinates of the plane are known, the 2D locations are detected, then the estimation of the projective parameters is called the $\mathrm{PnP}$ problem. Mathematically, the PnP optimization can be written as

$$
\arg \min _{R, t} \sum_{i=1}^{G_{x}} \sum_{j=1}^{G_{y}} \sum_{k=1}^{N} \operatorname{Rep}\left(R, t,\left[\begin{array}{c}
u_{i, \alpha} \\
v_{j, \alpha}
\end{array}\right],\left[\begin{array}{c}
x_{i, \alpha}^{\prime} \\
h
\end{array}\right]\right)
$$

where the definition of the function Rep is as follows:

$$
\begin{array}{r}
\operatorname{Rep}\left(R, t,\left[\begin{array}{l}
u_{i} \\
v_{j}
\end{array}\right],\left[\begin{array}{c}
x_{i}^{\prime} \\
y_{j}^{\prime} \\
h
\end{array}\right]\right)= \\
\left\|\operatorname{DeHom}\left(R\left[\begin{array}{c}
x_{i}^{\prime} \\
y_{j}^{\prime} \\
h
\end{array}\right]+t\right)-\left[\begin{array}{c}
u_{i} \\
v_{j}
\end{array}\right]\right\|_{2}^{2} .
\end{array}
$$

The applied comma (') means that the origin of the coordinate system for chessboard corners are placed at $\left[o_{x}, o_{y}\right]^{T}$. Function DeHom gives the dehomogeneous $2 \mathrm{D}$ vector of a spatial vector as $\operatorname{DeHom}\left([X, Y, Z]^{T}\right)=[X / Z, Y / Z]^{T}$.

There are solutions that can cope with planar points. We used the EPnP (Lepetit et al., 2009) algorithm for our approach. At this point, the relative transformation between the chessboard planes and the camera can be calculated. They are denoted by $\left[R^{1}, t^{1}\right]$, and $\left[R^{2}, t^{2}\right]$. The altitude of the chessboard can be measured. Without loss of generalization, altitude of the first plane can be set to zero: $h_{1}=0$. (The simplest way is to set the first chessboard to the turntable. Then the altitude of the second chessboard can be easily measured with respect to the turntable.)

The estimation of one parameter is relatively simple. We solve it by exhaustive search. The best value is given by the rotation for which the reprojection error of the PnP problem is minimal:

$$
\arg \min _{R, t} \sum_{i=1}^{G_{x}} \sum_{j=1}^{G_{y}} \sum_{k=1}^{N}\left(\operatorname{Rep}^{1}+\operatorname{Rep}^{2}\right)
$$

where

$$
\begin{gathered}
\operatorname{Rep} p^{1}=\operatorname{Rep}\left(R^{1}, t^{1},\left[\begin{array}{c}
u_{i, \alpha_{k}} \\
v_{j, \alpha_{k}}
\end{array}\right],\left[\begin{array}{c}
x_{i, \alpha_{k}}^{\prime} \\
y_{j, \alpha_{k}}^{\prime} \\
0
\end{array}\right]\right) \\
\operatorname{Rep}^{2}=\operatorname{Rep}\left(R^{2}, t^{2},\left[\begin{array}{c}
u_{i, k} \\
v_{i, k}
\end{array}\right],\left[\begin{array}{c}
x_{i, \alpha+\alpha_{k}}^{\prime} \\
y_{j, \alpha+\alpha_{k}}^{\prime} \\
h
\end{array}\right]\right)
\end{gathered}
$$

where the upper index denotes the number of the chessboard. The relationship between the left and right terms are that the spatial points have to rotated with the same angle, but a fix angular offset $\Delta \alpha$ has to be added to each rotation for the second chessboard plane with respect to the first one. (The setup is visualized in Fig. 3.) The impact of $\Delta \alpha$ for the second rotation matrix is written as follows:

$$
R^{2}=\left[\begin{array}{ccc}
\cos \Delta \alpha & -\sin \Delta \alpha & 0 \\
\sin \Delta \alpha & \cos \Delta \alpha & 0 \\
0 & 0 & 1
\end{array}\right] R^{1}
$$

The minimization problem is also a $\mathrm{PnP}$ one, therefore it can be efficiently solved by (Lepetit et al., 2009). The estimation of $\Delta \alpha$ is obtained by an exhaustive search.

Finally, the extrinsic parameters of the projector are computed by running the $\mathrm{PnP}$ algorithm again for the corners detected in the projector images. The obtained projector parameters have to be transformed by the inverse of the camera extrinsic parameters since our global coordinate system is fixed to the camera. 


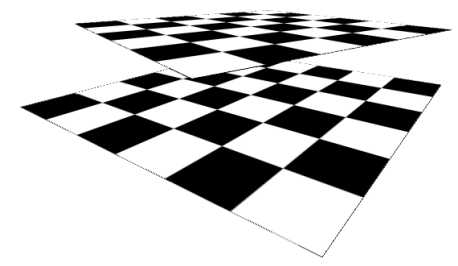

Figure 3: Visualized chessboard planed in the first position. The edged are not parallel, therefore the relative angle $\Delta \alpha$ has to be estimated.

\subsection{Object Reconstruction}

The object reconstruction looks very similar to the projector calibration. In this case, an object is placed on the turntable instead of the chessboard. Structured light is projected onto it, images are taken, then the object is rotated. This procedure is repeated until the object returns to the starting position. Then we decode the projector pixels back from the structured light projected in each image set. After it is done, we use the Hartley-Strum triangulation (Hartley and Sturm, 1997) for corresponding camera-projector pixels due to its accuracy to determine the object points from one viewpoint. We calculate these for each viewpoint, and then we can combine the point sets together, which results a $3 \mathrm{D}$ points et of the full object.

\section{RESULTS}

The main advantage of our method is that the whole GT data generation is totally automatic. Therefore, arbitrary number of objects can be reconstructed. We show here four typical objects that have well trackable feature points. They are as follows:

- Dinosaur. A typical researcher enjoys the reconstruction of dinosaurs as it is shown in several scientific papers, e.g ("Fitzgibbon et al., 1998). Fortunately, kids also like it, and one of the authors' sons has a plastic dinosaurs that could be reconstructed. Therefore, we inserted a dino to our testing dataset.

- Flacon. The Plastic holder is a good test case since at least one well-textured label is fixed on the surface of a usual flacon.

- Plush Dog. The tracking of the feature point of a soft toy is a challenging task as it does not have a flat surface. For this reason, we include a plush dog into the testing database.
- Poster. The last sequence of our dataset is a rotating newspaper page. It is useful since it is a simple textured plane. The efficiency of the trackers can be checked in this example due to two reasons: (i) there is no occlusion, and (ii) the feature tracking is the determination of a plane-plane homography.

During the test, the objects were rotated by the turntable, the difference of the degree of two subsequent was set to $3^{\circ}$. Our GT tracking data generator has two modes. (i) The first version regularly generates the feature points in the first image. The feature points are located across a regular grid in the valid region of the camera image. (ii) The points in the first image is determined by a feature generator. We use the SIFT features (Lowe, 1999) in our testing examples, but arbitrary feature generators can be included.

Then the generated feature points were reconstructed in the first image using the structured light. Then these 3D reconstructed point coordinates were rotated around the turntable axis with the known rotating axis, and projected to the next image. This procedure was repeated for all the images of the test sequence. The $2 \mathrm{D}$ feature coordinates after projection give the final GT for quantitative feature tracker comparison.

The input images of the sequences are visualized in Figs. $4-7$. The 3D model of the reconstructed objects are also visualized in these Figures except the Poster as it is a planar paper and its 3D model is not interesting. The 3D models are represented by colored point clouds, however, the color itself does not influence the reconstruction. It is only painted due to its spectacularity.

The computed ground truth data for the four examined sequences are pictured in Figs. 8-11. The first row shows the tracked points when the points are selected across a grid. The second rows of Figs. 8- 7 consist of images with the tracked GT SIFT feature points (yellow dots). We also applied an automatic feature tracker (BruteForceMatcher of OpenCV) and the estimated feature points are drawn in the images with red color. However, the comparison of feature vectors is out of the scope of this paper, we only want to demonstrate that this comparison can be easily carried out.

The obtained ground truth data were visually checked by us and we have not found any inaccuracy on it. We think that the accuracy is below pixel, in other word, subpixel accuracy was reached. This is extremely low as the camera resolution is $2592 \times 1936$ (5 Mpixel). 

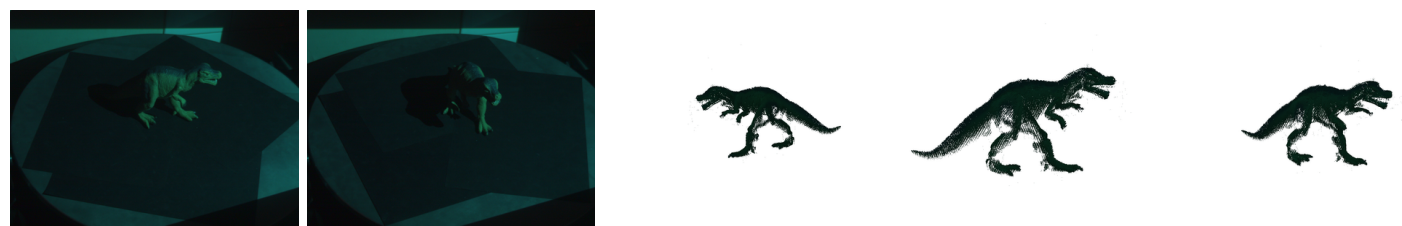

Figure 4: Two images of the 'Dino' sequence and the reconstructed 3D point cloud from three viewpoints.
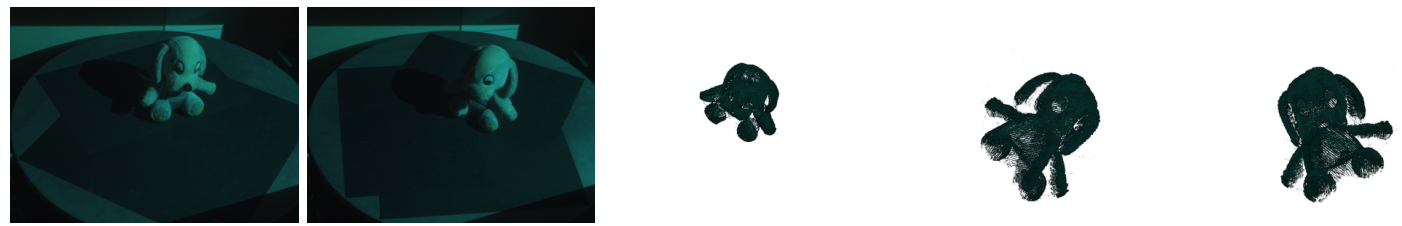

Figure 5: Two images of the 'Plush Dog' sequence and the reconstructed 3D point cloud from three viewpoints.
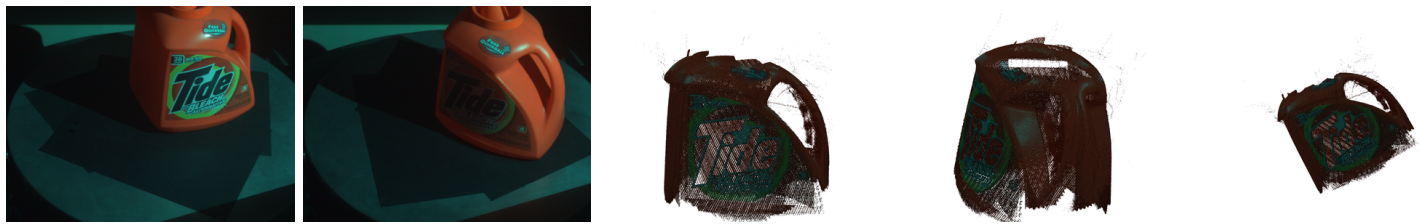

Figure 6: Two images of the 'Flacon' sequence and the reconstructed 3D point cloud from three viewpoints.
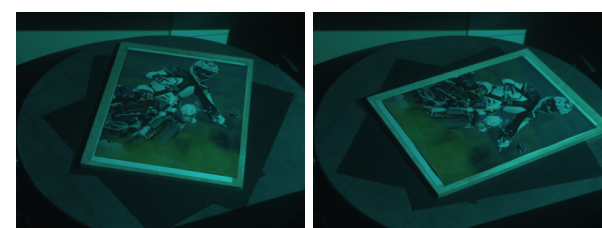

Figure 7: Two images from the 'Poster' sequence.

\section{COMPARISON OF WELL-KNOWN FEATURE TRACKERS}

Thought this paper does not concentrate on the comparison of feature trackers, we run the most popular trackers implemented in the OpenCV library ${ }^{3}$. Each tracker consists of separated methods in order to generate, describe, and finally track good features. However, the generation and description is given by the same algorithms in our examples. The tracking (matching) is different, we selected the most accurate tracker for the generators based on our tests.

The applied feature generators are as follows:

1. Scale Invariant Feature Transform (SIFT) (Lowe, 1999)

2. Speeded Up Robust Features (SURF) (Bay et al., 2008)

3. KAZE (Alcantarilla et al., 2012)

$\overline{{ }^{3} \mathrm{http}: / / \text { opencv.org }}$
4. Accelerate KAZE (AKAZE) (Alcantarilla et al., 2013)

5. Binary Robust Invariant Scalable Keypoints (BRISK) (Leutenegger et al., 2011)

6. ORB (Oriented FAST and Rotated BRIEF) (Rublee et al., 2011)

Several matchers have been tested for the selected feature detectors/descriptors. OpenCV supports a brute-force based matchers as well as the Flann (Fast Approximate Nearest Neighbor) matcher (Muja and Lowe, 2009). In the case of the brute-force based matchers, all descriptors on the first image are compared with all descriptors on the second image and the best match, when the distance of the descriptors is the lowest, is chosen. In Fig 12, 'BF_L1' means that the brute-force matcher is used with the $L_{1}$ norm, while 'BF H1' means that the brute-force matcher is used with the Hamming distance. $L_{2}$ norm is used for algorithms 'BF_L2' and 'BF_H2'.

The rival trackers are compared on every test sequence. The error of the feature tracking is defined as the difference between the tracked and GT coordinates. The averages are calculated for each frame, and this mean values is the error for the examined feature. Then the error of all feature errors are computed as well. The median of the feature errors is also calculated. These mean and median values of the rival trackers are visualized in the plots of Fig. 12.

The detailed description of the feature detectors and the evaluation of the test results are out of the 

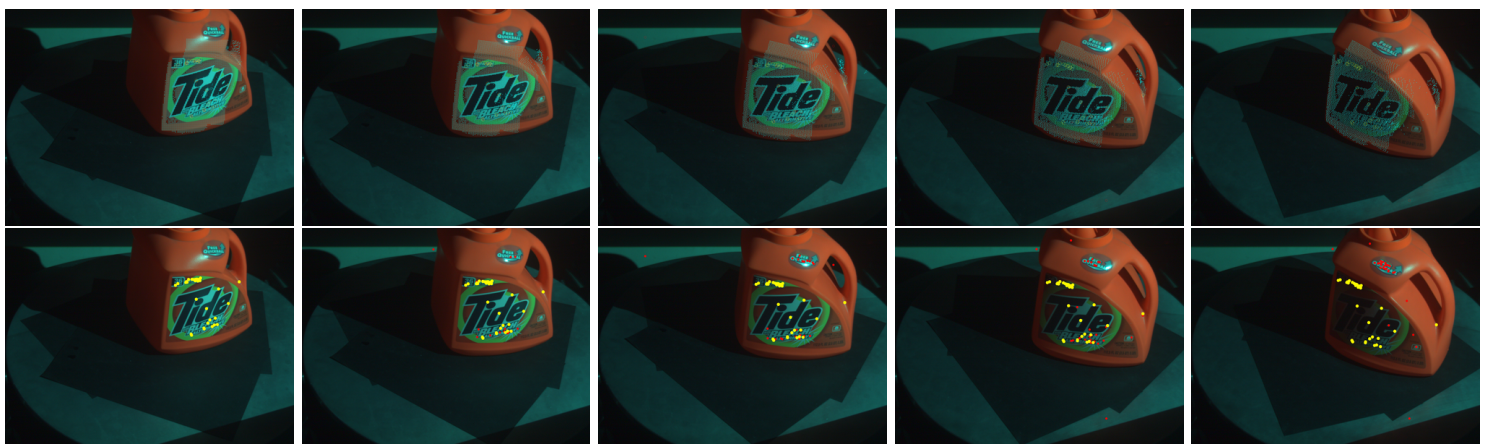

Figure 8: The visualized ground truth tracking data drawn on images of the 'Flacon' sequence. Top row: features generated by a grid within valid image region. Bottom row: features generated by SIFT method.
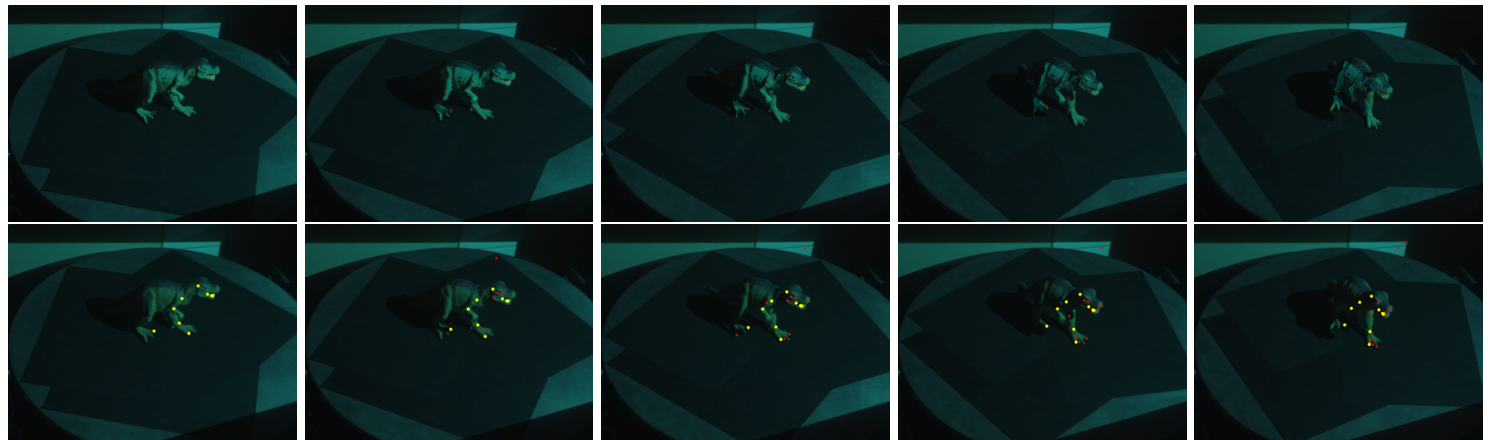

Figure 9: The visualized ground truth tracking data drawn on images of the 'Dino' sequence. Top row: features generated by a grid within valid image region. Bottom row: features generated by SIFT method.
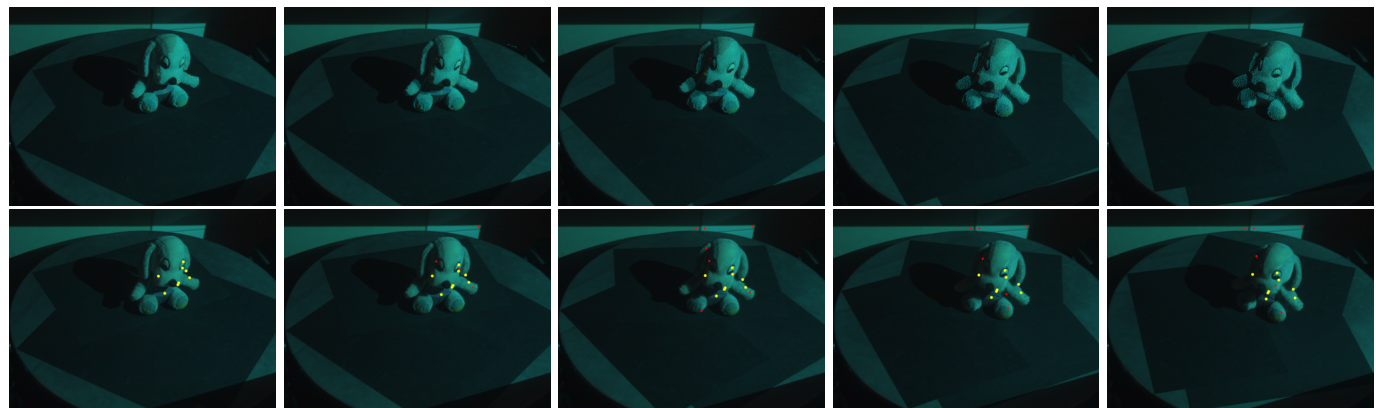

Figure 10: The visualized ground truth tracking data drawn on images of the 'Plush Dog' sequence. Top row: features generated by a grid within valid image region. Bottom row: features generated by SIFT method.
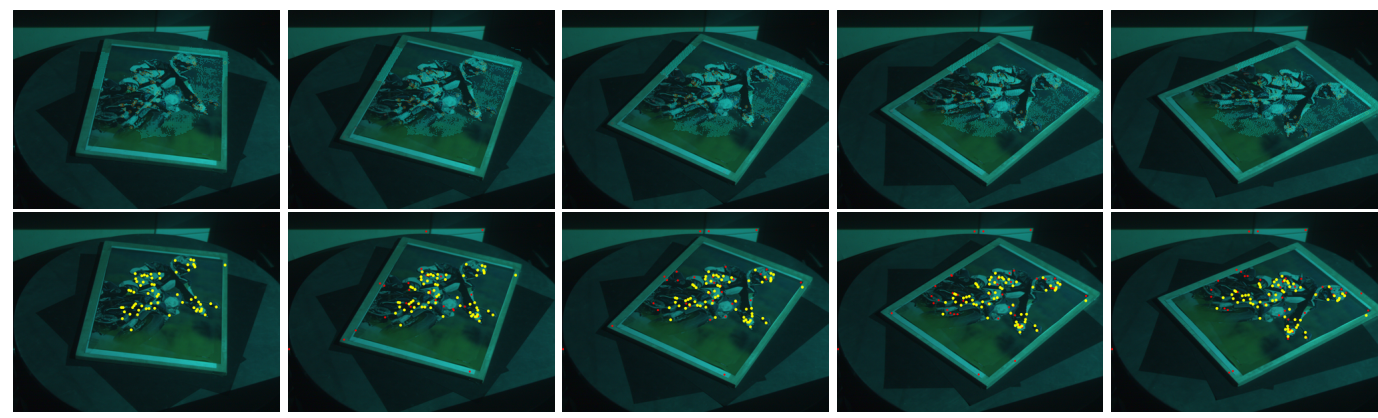

Figure 11: The visualized ground truth tracking data drawn on images of the 'Poster' sequence. Top row: features generated by a grid within valid image region. Bottom row: features generated by SIFT method. 
scope of this paper. We know that more information are required to compare the methods, this short description only wants to demonstrate that quantitative comparison is possible by our equipment. A deep comparison will be published very soon.
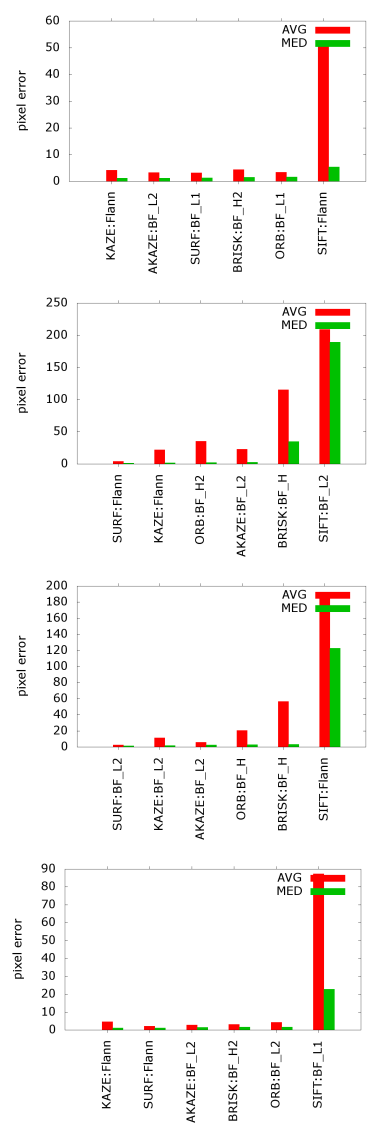

Figure 12: Tracking error for test sequences. Avg: mean, Med: median. From top to bottom: Results for 'Flacon', 'Dino', Plush Dog', and 'Poster' testing objects.

\section{LIMITATIONS \& FUTURE WORK}

The main goal of the approach proposed here is to be able to generate ground truth tracking data of real-world rotating objects. Therefore, the turntablebased equipment is unable to simulate moving cameras. However, other databases (e.g. the famous Middlebury one) can do that, thus our approach should be unified with existing datasets. Nevertheless, our equipment contains two moving arms for both the camera and projector, therefore novel viewpoints can be added to the system. It is possible if the arms are very accurately calibrated. This is a possible feature work of our GT generation project.
Another disadvantage of the current system is that part of the objects can be self-occluded due to the object rotation. This cannot be detected by the hardware, therefore surface reconstruction is required to detect if the part of the scanned 3D object is occluded by another part. To avoid this problem, we plan to develop a continuous surface reconstruction method for free-form spatial objects. If their quality is reliable, it will help to detect the self-occlusion of the moving objects.

\section{CONCLUSIONS}

We have proposed a novel GT tracking data generator here that can automatically produce very accurate tracking data of rotating real-world spatial objects. The main novelty of our approach is that it consists of a turntable, and we showed how this turntable can be accurately calibrated. Finally, the validation of our equipment was shown. It was justified that the proposed structured-light 3D scanner can produce accurate tracking data as well as realistic 3D point clouds. The GT tracking data are public, they are available at our web page ${ }^{4}$.

\section{REFERENCES}

Alcantarilla, P. F., Bartoli, A., and Davison, A. J. (2012). Kaze features. In ECCV (6), pages 214-227.

Alcantarilla, P. F., Nuevo, J., and Bartoli, A. (2013). Fast explicit diffusion for accelerated features in nonlinear scale spaces. In British Machine Vision Conf. $(B M V C)$.

Anwar, H., Din, I., and Park, K. (2012). Projector calibration for $3 \mathrm{~d}$ scanning using virtual target images. International Journal of Precision Engineering and Manufacturing, 13(1):125-131.

Audet, S.and Okutomi, M. (2009). A user-friendly method to geometrically calibrate projector-camera systems. In Computer Vision and Pattern Recognition Workshops, pages $47-54$.

Baker, S., Scharstein, D., Lewis, J., Roth, S., Black, M., and Szeliski, R. (2011). A database and evaluation methodology for optical flow. International Journal of Computer Vision, 92(1):1-31.

Bay, H., Ess, A., Tuytelaars, T., and Van Gool, L. (2008). Speeded-up robust features (surf). Computer Vision and Image Understanding, 110(3):346-359.

Björck, Å. (1996). Numerical Methods for Least Squares Problems. Siam.

Bradley, C., Vickers, G., and Tlusty, J. (1991). Automated rapid prototyping utilizing laser scanning and free-

\footnotetext{
${ }^{4}$ http://web.eee.sztaki.hu
} 
form machining. CIRP Annals - Manufacturing Technology, 41(1):437-440.

Fischler, M. and Bolles, R. (1981). RANdom SAmpling Consensus: a paradigm for model fitting with application to image analysis and automated cartography. Commun. Assoc. Comp. Mach., 24:358-367.

"Fitzgibbon, A. W., Cross, G., and Zisserman, A. ("1998"). "automatic 3D model construction for turn-table sequences". In "3D Structure from Multiple Images of Large-Scale Environments, LNCS 1506", pages "155170 ".

Gauglitz, S., Höllerer, T., and Turk, M. (2011). Evaluation of interest point detectors and feature descriptors for visual tracking. International Journal of Computer Vision, 94(3):335-360.

Hartley, R. I. and Sturm, P. (1997). Triangulation. Computer Vision and Image Understanding: CVIU, 68(2):146157.

Hartley, R. I. and Zisserman, A. (2003). Multiple View Geometry in Computer Vision. Cambridge University Press.

Jamil Draréni, Sébastien Roy, P. S. (2009). Geometric video projector auto-calibration. In Proceedings of the IEEE International Workshop on Projector-Camera Systems, pages 39-46.

Kazo, C. and Hajder, L. (2012). High-quality structuredlight scanning of 3D objects using turntable. In IEEE 3 rd International Conference on Cognitive Infocommunications (CogInfoCom), pages 553-557.

Lepetit, V., F.Moreno-Noguer, and P.Fua (2009). Epnp: An accurate $\mathrm{o}(\mathrm{n})$ solution to the pnp problem. International Journal Computer Vision, 81(2):155-166.

Leutenegger, S., Chli, M., and Siegwart, R. Y. (2011). Brisk: Binary robust invariant scalable keypoints. In Proceedings of the 2011 International Conference on Computer Vision, pages 2548-2555.

Liao, J. and Cai, L. (2008). A calibration method for uncoupling projector and camera of a structured light system. In IEEE/ASME International Conference on Advanced Intelligent Mechatronics, pages 770 - 774.

Lowe, D. G. (1999). Object recognition from local scaleinvariant features. In Proceedings of the International Conference on Computer Vision, ICCV '99, pages $1150-1157$.

Martynov, I., Kamarainen, J.-K., and Lensu, L. (2011). Projector calibration by "inverse camera calibration". In SCIA, volume 6688 of Lecture Notes in Computer Science, pages 536-544.

Moreno, D. and Taubin, G. (2012). Simple, accurate, and robust projector-camera calibration. In 2012 Second International Conference on 3D Imaging, Modeling, Processing, Visualization \& Transmission, Zurich, Switzerland, October 13-15, 2012, pages 464-471.

Muja, M. and Lowe, D. G. (2009). Fast approximate nearest neighbors with automatic algorithm configuration. In In VISAPP International Conference on Computer Vision Theory and Applications, pages 331-340.

Nayar, S. K., Krishnan, G., Grossberg, M. D., and Raskar, R. (2006). Fast separation of direct and global components of a scene using high frequency illumination. ACM Trans. Graph., 25(3):935-944.
Pal, C. J., Weinman, J. J., Tran, L. C., and Scharstein, D. (2012). On learning conditional random fields for stereo - exploring model structures and approximate inference. International Journal of Computer Vision, 99(3):319-337.

Park, S.-Y. and Park, G. G. (2010). Active calibration of camera-projector systems based on planar homography. In $I C P R$, pages 320-323.

Rublee, E., Rabaud, V., Konolige, K., and Bradski, G. (2011). Orb: An efficient alternative to sift or surf. In Proceedings of the 2011 International Conference on Computer Vision, ICCV '11, pages 2564-2571.

Sadlo, F., Weyrich, T., Peikert, R., and Gross, M. H. (2005). A practical structured light acquisition system for point-based geometry and texture. In Symposium on Point Based Graphics, Stony Brook, NY, USA, 2005. Proceedings, pages 89-98.

Scharstein, D., Hirschmüller, H., Kitajima, Y., Krathwohl, G., Nesic, N., Wang, X., and Westling, P. (2014). High-resolution stereo datasets with subpixel-accurate ground truth. In Pattern Recognition - 36th German Conference, GCPR 2014, Münster, Germany, September 2-5, 2014, Proceedings, pages 31-42.

Scharstein, D. and Szeliski, R. (2002). A Taxonomy and Evaluation of Dense Two-Frame Stereo Correspondence Algorithms. International Journal of Computer Vision, 47:7-42.

Scharstein, D. and Szeliski, R. (2003). High-accuracy stereo depth maps using structured light. In $C V P R$ (1), pages 195-202.

Seitz, S. M., Curless, B., Diebel, J., Scharstein, D., and Szeliski, R. (2006). A comparison and evaluation of multi-view stereo reconstruction algorithms. In 2006 IEEE Computer Society Conference on Computer Vision and Pattern Recognition (CVPR 2006), 17-22 June 2006, New York, NY, USA, pages 519-528.

Sturm, J., Engelhard, N., Endres, F., Burgard, W., and Cremers, D. ("2012"). "a benchmark for the evaluation of rgb-d slam systems". In "Proc. of the International Conference on Intelligent Robot Systems (IROS)".

Xu, Y. and Aliaga, D. G. (2007). Robust pixel classification for $3 \mathrm{~d}$ modeling with structured light. In Proceedings of the Graphics Interface 2007 Conference, May 2830, 2007, Montreal, Canada, pages 233-240.

Yamauchi, K., Saito, H., and Sato, Y. (2008). Calibration of a structured light system by observing planar object from unknown viewpoints. In 19th International Conference on Pattern Recognition, pages 1-4.

Zhang, Z. (2000). A flexible new technique for camera calibration. IEEE Transactions on Pattern Analysis and Machine Intelligence, 22(11):1330-1334. 